EUROPEAN JOURNAL OF ENVIRONMENT

AND

PUBLIC HEALTH
European Journal of Environment and Public Health,

2018, 2(1), 01

ISSN: $2468-1997$

\title{
Nursing Associated Medication Errors: Are Internationally Educated Nurses Different from U.S. Educated Nurses?
}

\author{
Jay J. Shen ${ }^{1 *}$, Alona D. Angosta ${ }^{2}$, Michelle Sotero ${ }^{1}$, Jennifer Rice ${ }^{1}$, Karmjit Raju ${ }^{3}$ \\ ${ }^{1}$ Department of Health Care Administration and Policy, University of Nevada, Las Vegas, UNITED STATES \\ 2 School of Nursing, University of Nevada, Las Vegas, UNITED STATES \\ ${ }^{3}$ Nevada State College, University of Nevada, Las Vegas, UNITED STATES
}

*Corresponding Author: jay.shen@unlv.edu

Citation: Shen, J. J., Angosta, A. D., Sotero, M., Rice, J. and Raju, K. (2018). Nursing Associated Medication Errors: Are Internationally Educated Nurses Different from U.S. Educated Nurses?. European Journal of Environment and Public Health, 2(1), 01. https://doi.org/10.20897/ejeph/85002

Published: February 28, 2018

\begin{abstract}
Medication errors can be detrimental to patient safety and contribute to additional costs in healthcare. The United States has seen a steady increase in internationally-educated nurses (IENs) entering the nursing workforce. The current study builds upon the existing research examining the relationship between IENs and medication errors by controlling for confounding factors and testing whether IENs were more likely to make multiple medication errors compared to USENs. This study was a quasi-case control study. The 2006 and 2010 medication error incident data from hospital risk management departments were used. The final sample was 1,773, representing 788 registered nurse in the case group and 985 registered nurses in the control group. Multivariable analyses were conducted to examine single medication error, multiple errors, and consequence of medication errors, in comparing the IENs to USENs. IENs tended to have multiple errors more often than USENs in $2006(31.7 \%$ for IENs and $20.5 \%$ for USENs, $p=0.03)$, but these differences became marginally significant after combining both years of data and completing the multivariable models adjusting for covariates (Odds ratio $=1.38, \mathrm{p}=0.06$ ). No significant differences in making a single error and medication error consequences were observed between IENs and USENs. Although no significant differences between IENs and USENs in having medication error incidents were observed, IENs might be more likely to have multiple medication error incidents in a year compared to USENs. Policies that encourage targeted orientation addressing implicit belief systems about the nursing role and explains patient safety expectations as well as procedures for medication administration may be beneficial for IENs. Supportive leadership that is culturally competent, ensures ongoing continuing education in pharmacology, and provides culturally appropriate incentives for self-reporting medication errors are important.
\end{abstract}

Keywords: medication error, internationally educated nurse

\section{INTRODUCTION}

Medication errors affect patient safety and contribute to additional healthcare costs. According to the United States Food and Drug Administration (2009), medication errors are defined as any preventable event that may cause or has caused patients harm. Similarly, an adverse drug event (ADE) is defined as harm experienced by a patient as a result of exposure to a medication. Medication errors are widespread in the United States, and ADEs account for as many as 1.5 million incidences each year (Institute of Medicine [IOM], 2006). Close to 5\% of patients in hospital settings are affected by ADEs, making this one of the most common types of medical errors (Agency 
for Healthcare Research and Quality, 2012). According to the Food and Drug Administration (2016), "medication errors cause at least one death every day and injure approximately 1.3 million people annually." Studies also suggest many medication errors among nurses are rarely reported at clinical settings (Bayazidi et al., 2012; Ehsani et al., 2013; Mayo and Duncan, 2004). Therefore, the true number of medication errors may be much higher than the ones actually reported.

Although medication errors could occur at all stages of medication use, they are frequently seen in the administration stage, for which nurses are responsible (IOM, 2006). While not all medication errors result in actual harm, those that do can be costly. Additional hospital incurred costs for preventable ADEs alone were an estimated $\$ 3.5$ billion in 2006 (IOM, 2006). Preventable ADEs among ambulatory Medicare enrollees cost $\$ 887$ million annually (IOM, 2006).

The number of internationally educated nurses (IENs) has steadily grown, and IENs have become a substantial part of the United States (U.S.) nursing workforce. Over the past few decades, in response to concerns about the growing demand for U.S. health care services and cyclic shortages of nurses, many U.S. healthcare providers with supportive immigration policies have turned to IENs to fill vacant nursing positions (Aiken et al., 2004; Polsky et al., 2007). The countries who have supplied the most IENs to the U.S. include the Philippines, Canada, United Kingdom, and India (Masselink and Jones, 2014). Consequently, the proportion of foreign born nurses increased dramatically, now making up one third or 155,000 of the 476,000 full-time registered nurses (RNs) in the U.S., with the majority working in inpatient settings (Buerhaus et al., 2009). In 2008, the number of IENs in the U.S. tripled since the 1970s, to 165,000 (Masselink and Jones, 2014). IENs tend to work more hours annually than U.S. educated nurses (USENs) and they are more likely to work in complex care settings such as intensive care unit (ICU), and stepdown/ transitional units (Xu et al., 2010).

The process for IENs to be licensed as a nurse and practice in the U.S. is rigorous and ensures that they meet the same standards as the USENs. Just like USENs, IENs must pass the National Council Licensure Examination for Registered Nurses (NCLEX-RN) in order to practice in the U.S. Prior to applying to take the NCLEX-RN, most state nursing boards required IENs to have a prescreening service by the Commission on Graduates of Foreign Nursing Schools (CGFNS), which includes: a credential verification pertaining to the U.S. equivalent nursing education from non-U.S. programs, passing a nursing knowledge test, and passing an English proficiency test (Davis and Nichols, 2002). The NCLEX-RN passing rates of IENs have dramatically improved from $15-20 \%$ before the CGFNS certificate program in the 1970s to $85-90 \%$ in the early 2000s (David and Nichols, 2002). More than $80 \%$ of CGFNS applicants in 2000 held bachelor's degrees (Davis and Nichols, 2002). A higher percentage of IENs received their basic nursing education in a baccalaureate nursing programs than USENs (Shen et al., 2014).

Despite the increasing numbers and higher educational levels of IENs, they struggle with distinct challenges during their integration into the U.S. healthcare system. These challenges include language barriers/fluency, cultural dissimilarities, and uncertainty of new nursing roles/expectations reported by prior studies (Davis and Nichols, 2002; Lin, 2014; Tregunno et al., 2009; Shen et al., 2013; Yahes and Dunn, 1996).

IENs who learned formal English as a second language in their native country may experience problems with linguistic performance and accent, as well as with differences in dialect and communication styles of native English speakers in the health care setting. (Shen et al., 2012; Xu et al., 2013; Xu et al., 2010; Shen et al., 2010). IENs may also experience differences in cultural norms for expressing nonverbal communication such as hugging, lowering their body position to a patient's level, leaning forward, shaking hands, and therapeutic touch when interacting with patients (Xu et al., 2012). Two important cultural dimensions that predict workplace safety are respect for authority and individualism-collectivism (Casey et al., 2015). IENs adhering to cultural norms that readily accept and defer to centralized authority may have difficulty openly communicating to supervisors about needed training that would prevent medication errors and near misses (Sherwood and Shaffer, 2014; Marx, 2014). Collectivist cultural values prioritize the well-being of the group and relationships. For some IENs, pointing out errors made by a member of the health care team may cause embarrassment or conflict with the team goal of "exemplary safety outcomes" (Casey, et al., 2015). Perceived and actual workplace discrimination may also contribute to poor communication, less supportive relationships with leadership, lower levels of workplace integration and feelings of psychological safety for IENs. (Sherwood and Shaffer, 2014; Casey, et al., 2015) Finally, IENs may experience uncertainty in the practice setting due to differences in terminology, medication names, conversion of weights and measures, and organizational protocols and procedures (Neff and Harman, 2013). These challenges pose potential threats to patient safety and quality of care (Xu, 2007). Despite passing the NCLEX-RN and the standardized English language tests, these two criteria do not predict success with the communication techniques and style necessary for working with nursing colleagues, health care providers, hospital staff, and patients (Shen et al., 2010).

The national policy priority of improving patient safety and quality in healthcare delivery, reflected by the National Academy of Health's landmark books, To Err Is Human: Building a Safer Health System (2000), Crossing the Quality Chasm (2001), and Preventing Medication Errors (2006), establishes the expectation that internationally educated nurses are anticipated to share more tasks with USEN. To date, scientifically based comparison studies focusing 
on medication errors of IENs are rare, despite an alarmingly high prevalence of medication errors and a large number of IENs working in the U.S. There has been one study so far that compares medication error report data in a hospital setting to medication error incident rates between IENs and USENs (Shen et al., 2015). This study, using medication error incident as the unit of analysis, found no significant differences in medication errors between IENs and USENs in 2010. However, this study focused on medication error incidents without controlling for potential confounding factors and did not consider multiple errors being made by the same nurse. The current study builds upon the existing research examining the relationship between IENs and medication errors by controlling for confounding factors and testing whether IENs were more likely to make multiple medication errors compared to USENs.

\section{METHODS}

This study used a retrospective, quasi-case control design for this study. The unit of analysis was the registered nurse (RN) through which multiple errors by the same nurse could be examined. The 2006 and 2010 data were collected at five short-term acute hospitals in the Southwest region of the U.S. The data collection began in November 2011 and was completed on July 2014 by data collectors hired by each of the hospitals, respectively. The data collectors were trained before they used the Microsoft Access data collecting tool. We obtained medication error information from the risk management departments and information about RNs' demographics and educational background from the participating hospitals' Human Resources department.

A case was defined as an incident in which an $\mathrm{RN}$ made an error during medication administration. RNs in the case group were randomly selected from the medication error case list. If the number of cases was not sufficient to conduct the random sampling at a hospital in a specific year, all cases in that year were selected. RNs in the control group were randomly selected from the human resource department among RNs who did not make medication errors in specific years.

The sample size calculation was detailed elsewhere (Shen et al., 2015), and the minimum sample size of 816 per year (Fleiss et al., 2003) was achieved. The 2010 case group was oversampled for the purpose of investigating potentially more severe, but rare medication errors. 1,276 medication error incidents were selected for the case group, and 1,060 nurses were selected for control incidents. The original definition of a foreign educated nurse (IEN) was a nurse who was born outside of the U.S. and received his or her basic nursing education outside the U.S. However, after data collection, we found that HR records for about $40 \%$ of the RNs did not indicate the country of birth. As a result, we redefined the IEN. Our inclusion criteria for IENs were nurses who attended high school outside the U.S. and received their basic nursing education outside the U.S. We excluded RNs who attended high school in the U.S. and received basic nursing education in another country, RNs who attended high school in another country but received basic nursing education in the U.S., and RNs whose education information was missing. A total of 158 RNs were excluded and labeled as not meeting the criteria for IENs. Therefore, our final sample for data analysis was 1,773, representing $788 \mathrm{RNs}$ (the same nurse might have multiple medication error incidents in a year) in the case group and 985 nurses in the control group. In addition, we did not find any differences in the results after including and excluding RNs from Canada whose primary language is English from the sample for data analysis.

Several key variables were formed and analyzed. A dummy variable of IEN was created to indicate whether an $\mathrm{RN}$ was an IEN (with a value of 1 ) or a U.S.-educated nurse (with a value of 0 ). Percentages of IENs and USENs were compared between the case group and the control group. Comparisons between the IENs and USENs were made in regard to having multiple medication incidents and severity of medication errors. A dummy variable of Multiple Error was created with a value of " 1 " indicating a RN having more than one medication error incidents and a value of " 0 " indicating one incident. The severity of medication errors was categorized according to the following: (1) errors that did not reach patients, (2) errors that reached patients but did no harm, (3) Errors that reached patients and resulted in increased monitoring of the patient, and (4) errors that reached patients and caused harm. An ordinal variable of Med_Err_Consequence with values from 1 to 4 was created to reflect the four levels of the severity from the least severe (1) to most severe (4). No medication error caused the death of the patient in the sample.

Several multivariable techniques were applied in data analysis depending on the type of the dependent variable in the model. The random effect logistic regression was used when the IEN and Multiple Error were dependent variables, respectively, while the hospital served as the random effect. The generalized ordinal logistic regression was applied when Med_Err_Consequence was the dependent variable, while the hospital served as the random effect. Covariates were taken into account in the data analysis. They included the sex, age, level of basic nursing education (i.e., associate degree versus bachelor degree), highest education level (i.e., associate degree, bachelor degree and advance degree), length of nursing experience ( $<1$ year, $1-9$ years, $10-19$ years, $>=20$ years), parttime versus full-time, change in work unit while the error occurred (yes or no), nurse's regular work unit (e.g., 
Table 1. Nurses' Demographic, Educational, and Working Characteristics

\begin{tabular}{|c|c|c|c|c|}
\hline \multirow[b]{2}{*}{ Variable } & \multicolumn{2}{|c|}{ Case } & \multicolumn{2}{|c|}{ Comparison } \\
\hline & $\begin{array}{c}\text { IEN } \\
(\mathrm{n}=227)\end{array}$ & $\begin{array}{c}\text { USEN } \\
(n=561)\end{array}$ & $\begin{array}{c}\text { IEN } \\
(\mathrm{n}=292)\end{array}$ & $\begin{array}{c}\text { USEN } \\
(n=693)\end{array}$ \\
\hline Age, year, mean (St.D.) & $41.8(8.8)$ & $40.4(11.7)$ & $43.2(8.9)$ & $40.9(11.3)$ \\
\hline Female & $86.8 \%$ & $90.0 \%$ & $91.1 \%$ & $85.4 \%$ \\
\hline \multicolumn{5}{|l|}{ Basic nursing education* } \\
\hline Associate Degree & $4.9 \%$ & $48.5 \%$ & $4.1 \%$ & $50.1 \%$ \\
\hline Licensed Practiced Nurse (LPN) & $0.4 \%$ & $7.3 \%$ & $0.3 \%$ & $5.9 \%$ \\
\hline BS in Nursing & $85.5 \%$ & $39.0 \%$ & $88.0 \%$ & $40.5 \%$ \\
\hline Diploma & $6.2 \%$ & $4.3 \%$ & $6.5 \%$ & $3.0 \%$ \\
\hline Advanced nursing (ADN) & $0.0 \%$ & $0.2 \%$ & $0.0 \%$ & $0.0 \%$ \\
\hline Unknown & $3.0 \%$ & $0.7 \%$ & $1.0 \%$ & $0.4 \%$ \\
\hline With advanced degree & $5.3 \%$ & $2.1 \%$ & $7.6 \%$ & $3.3 \%$ \\
\hline \multicolumn{5}{|l|}{ Type of employment* } \\
\hline Full-time hospital employee & $89.8 \%$ & $86.6 \%$ & $82.4 \%$ & $79.7 \%$ \\
\hline Part-time hospital employee & $6.2 \%$ & $6.5 \%$ & $9.3 \%$ & $5.3 \%$ \\
\hline On call employee & $1.3 \%$ & $2.7 \%$ & $4.1 \%$ & $9.2 \%$ \\
\hline Agency / travel nurse & $0.4 \%$ & $0.4 \%$ & $0.0 \%$ & $0.2 \%$ \\
\hline Unknown & $2.2 \%$ & $3.9 \%$ & $4.1 \%$ & $5.7 \%$ \\
\hline \multicolumn{5}{|l|}{ Working at current hospital* } \\
\hline Less than 1 year & $14.1 \%$ & $12.5 \%$ & - & - \\
\hline $1-10$ years & $80.2 \%$ & $77.9 \%$ & - & - \\
\hline $11-20$ years & $3.5 \%$ & $7.0 \%$ & - & - \\
\hline$>=21$ years & $1.3 \%$ & $2.5 \%$ & - & - \\
\hline Unknown & $0.90 \%$ & $0.20 \%$ & - & - \\
\hline
\end{tabular}

emergency department, intensive care, medical surgery), and year (2010 versus 2006). The STATA13.1 software was used for data analysis. This study obtained ethical approval from a university's Institutional Review Board and the Western Institutional Review Board. All identifiable information including name, social security number, and employee identification number was removed at the data collection sites by the hospitals before data were provided to the research team.

\section{RESULTS}

The sample's demographic, educational, and working characteristics by case/control group and by IEN/USEN are shown in Table 1. The average ages of RNs were from 40 to 43 years old across the four groups. Much higher percentages of IENs received a bachelor's degree in their basic nursing education than the USENs $(85.5 \%$ for IENs versus $39.0 \%$ for USENs in the case group and $88.0 \%$ for IENs and $40.5 \%$ for USENs in the control group). In terms of having an advanced degree, $5.3 \%$ of IENs and $2.1 \%$ of USENs in the case group had advanced degrees, while $7.6 \%$ in IENs and $3.3 \%$ in the control group had advanced degrees.

The percentages of IENs in both the case and control groups in 2006 were virtually identical and so were the percentages of IENs in the case and control groups in 2010 (Table 2). The results also indicate that about three quarters of the IENs came from the Philippines.

Percentages of having multiple medication error incidents as well as consequences of the medication error incidents among IENs and USENs, respectively, are displayed in Table 3. It seems that IENs tended to have multiple errors more often than USENs in 2006 (31.7\% for IENs and 20.5\% for USENs, $p=0.03$ ), but these differences were not statistically significant in 2010. In terms of the consequence of medication errors, no significant differences were found in 2006. However, significant differences were found in the consequences of medication error incidents between IENs and USENs in $2010(p=0.03)$. It appears that IENs tended to have more incidents with "Errors that did not reach patients" (22.5\% vs $15.8 \%)$ and were almost twice as likely to have "Errors that reached patients and caused harm" (13.3\% vs 7.9\%), and were likely to have fewer incidents with medication errors that reached patients but did no harm, or errors that reached patients resulting in increased monitoring. 
Table 2. Nurses' Basic Nursing Education Place by Group

\begin{tabular}{|c|c|c|c|c|c|c|}
\hline & \multicolumn{3}{|c|}{2006} & \multicolumn{3}{|c|}{2010} \\
\hline & Case $(n=276)$ & Control $(n=358)$ & p-Value & Case & Control & p-Value \\
\hline Nursing Education & & & 0.97 & & & 0.66 \\
\hline USEN & $190(68.8 \%)$ & $246(68.7 \%)$ & & $371(72.4 \%)$ & $447(71.3 \%)$ & \\
\hline IEN* & $86(31.2 \%)$ & $112(31.3 \%)$ & & $141(27.6 \%)$ & $180(28.7 \%)$ & \\
\hline Total & $276(100.0 \%)$ & $358(100.0 \%)$ & & $512(100.0) \%$ & $627(100.0 \%)$ & \\
\hline \multicolumn{7}{|l|}{$\overline{I E N}$} \\
\hline - Philippine & $70(25.4)$ & $89(24.9)$ & & $108(21.1)$ & $152(24.2)$ & \\
\hline - India & $6(2.2)$ & $6(1.7)$ & & $11(2.2)$ & $14(2.2)$ & \\
\hline - Canada & $3(1.1)$ & $6(1.7)$ & & $8(1.6)$ & $5(0.8)$ & \\
\hline - Other countries & $7(2.5)$ & $11(3.1)$ & & $14(2.8)$ & $9(0.6)$ & \\
\hline Total* & $86(31.6 \%)$ & $112(31.2 \%)$ & & $141(27.6 \%)$ & $180(28.7 \%)$ & \\
\hline
\end{tabular}

* The percentage in some column may vary slightly due to rounded data.

Table 3. Multiple Medication Error Incidents and Consequences of the Incidents: IENs versus USENs

\begin{tabular}{|c|c|c|c|}
\hline & IEN & USEN & p-Value \\
\hline \multicolumn{4}{|l|}{ Having multiple medication error incidents } \\
\hline$-2006(n=294)$ & & & 0.03 \\
\hline 1 incident & $71(68.3)$ & $151(79.5)$ & \\
\hline$>=2$ incidents & $33(31.7)$ & $39(20.5)$ & \\
\hline$-2010(n=549)$ & & & 0.15 \\
\hline 1 incident & $127(72.2)$ & $290(77.8)$ & \\
\hline$>=2$ incidents & $49(27.8)$ & $83(22.3)$ & \\
\hline \multicolumn{4}{|l|}{ Consequence of medication error incident } \\
\hline$-2006(n=292)$ & & & 0.56 \\
\hline Error not reach to patients & $7(6.8)$ & $22(11.6)$ & \\
\hline Error reached patients but no harm & $73(70.9)$ & $122(64.6)$ & \\
\hline Error reached patients with increased monitoring & 13(12.6) & $26(13.8)$ & \\
\hline Error reached patients with harm & $10(9.7)$ & 19(10.1) & \\
\hline$-2010(n=540)$ & & & 0.03 \\
\hline Error not reach to patients & $39(22.5)$ & $58(15.8)$ & \\
\hline Error reached patients but no harm & $92(53.2)$ & $235(64.0)$ & \\
\hline Error reached patients with increased monitoring & $19(11.0)$ & $45(12.3)$ & \\
\hline Error reached patients with harm & $23(13.3)$ & $29(7.9)$ & \\
\hline
\end{tabular}

Table 4. Relationships between IENs and Medication Errors $(n=1,889)$

\begin{tabular}{lccc}
\hline \multicolumn{1}{l}{ Odds Ratio } & $\mathbf{9 5 \%}$ CI & p-Value \\
\hline Being in Medication Etror Case group & & & \\
\hline IEN (Reference - USEN) & 1.03 & {$[0.83,1.28]$} & 0.76 \\
\hline Age & 0.99 & {$[0.98,1.00]$} & 0.07 \\
\hline Male & 0.83 & {$[0.62,1.10]$} & 0.20 \\
\hline Basic nursing education with a bachelor degree & 0.91 & {$[0.74,1.12]$} & 0.37 \\
\hline Advanced degree* & 0.68 & {$[0.41,1.12]$} & 0.13 \\
\hline Part-time RN & 0.63 & {$[0.48,0.81]$} & $<0.01$ \\
\hline Year of 2010 & 1.27 & {$[1.04,1.55]$} & 0.02 \\
\hline
\end{tabular}

*Advanced degree: master's or higher degrees

CI: Confidence Interval

Results from the multivariable analysis indicate no meaningful differences found between USENs and IENs in the odds of being in the medication error case group compared to the control group (Odds Ratio (OR) [95\% confidence interval $(\mathrm{CI})], 1.03[0.83,1.28], p=0.76)$. Overall, nurses who worked part-time were less likely to be in the medication error case group compared to those who worked full-time (OR 0.63 , CI $[0.48,0.81], \mathrm{p}=<0.01$ ) (Table 4).

Results from the multivariable analysis revealed the relationships between IENs and making multiple medication errors and consequences of the errors, respectively (Table 5). As compared to USENs, IENs were marginally more likely to make multiple medication errors, but this result was not statistically significant (OR [CI], $1.38[0.98,1.94], p=0.06)$. Emergency department nurses were marginally significantly less likely to make multiple medication errors than those in other work units (OR 0.44, CI [0.19-1.00], $p=0.05$ ). No differences in consequence of medication errors between IENs and USENs were observed. Interestingly, medication errors made in 2010 were less likely to have relatively more severe consequences as opposed to those in 2006 (OR [CI], $0.72[0.54$, $0.96], p=0.03)$. 
Table 5. Relationships between IENs and Having Multiple Medication Errors Incidents

\begin{tabular}{|c|c|c|c|}
\hline & Odds Ratio & $95 \% \mathrm{CI}$ & p-Value \\
\hline \multicolumn{4}{|c|}{ Making Multiple Errors $(n=835)$} \\
\hline IEN & 1.38 & {$[0.98,1.94]$} & 0.06 \\
\hline Age & 1.00 & {$[0.99,1.02]$} & 0.98 \\
\hline Male & 1.04 & {$[0.62,1.77]$} & 0.87 \\
\hline Advanced degree* & 0.70 & {$[0.25,1.96]$} & 0.50 \\
\hline Part-time RN & 0.66 & {$[0.38,1.14]$} & 0.14 \\
\hline \multicolumn{4}{|l|}{ Work unit } \\
\hline - Surgery & 1.38 & {$[0.87,2.19]$} & 0.17 \\
\hline - Intensive care unit (ICU) & 1.15 & {$[0.72,1.84]$} & 0.56 \\
\hline - Emergency department & 0.44 & {$[0.19,1.00]$} & 0.05 \\
\hline Year of 2010 & 0.90 & {$[0.63,1.28]$} & 0.55 \\
\hline \multicolumn{4}{|c|}{ Medication error consequences $(n=835)$} \\
\hline IEN & 0.97 & {$[0.72,1.31]$} & 0.86 \\
\hline Age & 1.00 & {$[0.99,1.01]$} & 0.76 \\
\hline Male & 1.04 & {$[0.67,1.62]$} & 0.86 \\
\hline Advanced degree* & 0.92 & {$[0.41,2.03]$} & 0.83 \\
\hline Part-time RN & 0.88 & {$[0.58,1.33]$} & 0.55 \\
\hline \multicolumn{4}{|l|}{ Work unit } \\
\hline - Surgery & 1.15 & {$[0.81,1.63]$} & 0.43 \\
\hline - Intensive care unit (ICU) & 1.41 & {$[0.97,2.05]$} & 0.07 \\
\hline - Emergency department & 1.12 & {$[0.66,1.89]$} & 0.68 \\
\hline Year of 2010 & 0.72 & {$[0.54,0.96]$} & 0.03 \\
\hline
\end{tabular}

*Advanced degree: master's or higher degrees

CI: Confidence Interval

\section{DISCUSSION}

Registered nurses (RNs) play a critical role in patient safety and health care quality. Exploring risk factors that contribute to medication errors and adverse drug events by nurses is crucial in reducing medication errors and decreasing morbidity and mortality in hospitals (Parry et al., 2015). Our study examined if internationally-educated nurses (IENs) were more likely to make multiple medication errors compared to their U.S.-educated nurses (USENs) and if there was any difference in the severity of medication errors.

Despite using multivariable analytical techniques for data analysis, we found consistent results with those of prior studies (Shen et al., 2015, Neff and Harman, 2013). Our findings showed no significant differences between IENs and USENs in making medication errors in the hospitals. However, logistic regression analysis, adjusting for covariates, indicated that IENs were marginally more likely to have multiple medication error incidents in a year compared to USENs. This result approached statistical significance $(p=0.064)$ and requires further investigation. Because IENs are more likely to work in complex care units with higher patient acuity, such as surgery, ICU, and transitional care, they may be at increased risk for making multiple medication errors (Keers et al., 2013). Systematic reviews of medication administration errors indicate that slips and lapses (e.g., misidentification of medication, misreading labels, prescriptions, or other documentation, or confusing look-a-like or sound-alike medications) due to distractions and interruptions are key contributors of medication errors (Keers et al., 2013; Parry et al., 2015). IENs who are also navigating language and cultural differences in the workplace may have higher rates of slips and lapses leading to multiple medication errors. Work place discrimination may also play a role in medication errors. Baptiste (2015) reports that internationally educated nurses face workplace discrimination. They tend to have more patients compare to other nurses. This increase in nurse-patient ratios can lead to stress, which leads to medical errors particularly medication errors (Hayes et al. 2012).

Finally, IENs tend to work more hours compared to USENs. Many IENs worked at more than one hospital (based on our anecdotal conversations with nursing administrators during the study process). Fatigue may play a role in higher multiple medication errors by IENs.

As noted above, the complexity of the work unit is a contributing factor for the occurrence of multiple medication errors. Our finding that nurses working in the emergency department are less likely to have multiple medication errors compared to other work units is not supported by previous research. Up to $14 \%$ of medication errors occur in the emergency department and represent approximately 3\% of all hospital-related medical errors (Weant et al., 2014). More research is needed to validate this finding. It is possible that nurses in the E.D.s involved in this study receive support that reduces the occurrence of multiple medication errors compared to other work units. For example, one study found that decentralized, full-time pharmacists deployed to the Emergency 
Department were able to observe and intervene to intercept medication errors and provide regular education sessions that reduced medication errors (Patanwala et al., 2010).

Although no significant difference between IENs and USENs regarding the consequence of medication errors was observed, we did find that the severity of medication error consequences was significantly less in 2010 than in 2006. There are several explanations. First, in 2006, the IOM published guidelines for preventing medication errors. Hospital implementation of these guidelines as well as advances in technology such as bar-coding systems and computerized provider-order entry systems (CPOE) between 2006 and 2010 may have contributed to the decrease in medication errors. Second, both IENs and USENs are required to take classes in clinical pharmacology. Recent focus on continuing education to increase knowledge of new drugs, dosing and administration available in the hospitals may have contributed to fewer medication errors in 2010 (Shen et al., 2015; Weant et al., 2014). Notably, however, one study found that nurses from non-Western birthplaces scored significantly lower on knowledge of pain management and use of analgesic medications compared to nurses educated in Canada or Britain (WattWatson et al., 2001). Another study found that Filipino IENs exhibited significantly lower quality of care in pain management in nursing homes compared to non-Filipino IENs and USENs (Wagner et al., 2015). It is important to research variations in knowledge about pharmacology among nursing populations since medication errors are so critical to patient safety. Hospitals may want to publish their interventions that increase pharmaceutical knowledge of nurses, particularly IENs, so others may adopt best practices in their own facilities.

Our findings also indicate that part-time nurses make fewer medication errors than their fulltime counterparts. One explanation may be that the part-time nurses work with less stress and psychological pressure. Another explanation is that the part-time nurses work fewer hours, which makes them less fatigued and not as burdened by the often heavy nursing workload. However, one study found that fulltime nurses actually had a lower chance of making a medication error compared to part-time nurses (Patanwala et al., 2010). The inconsistency between our findings and this study indicate further research is needed on comparing fulltime and part-time nurses in regard to making medication errors.

\section{IMPLICATIONS FOR NURSING PRACTICE, RESEARCH, AND POLICY}

IENs and USENs face similar challenges in the workplace resulting in medication errors. These consist of both individual level and organizational level factors. There is a dearth of research on IENs and quality of care outcomes in hospitals. This study adds important information to the body of literature. More research is merited given the challenges that IENs experience and the overall high incident rates of medication administration errors. Future studies might examine quality of care difference between IENs from Western, English speaking countries (e.g., U.K., Canada and Australia) compared to Non-Western countries (e.g., Philippines, India, Europe) to determine if subsets of IENs are at increased risk for medication errors. Research should also examine the impact of organizational communication and cultural norms on IENs transition and knowledge about patient safety and quality of care. A concern does exist about the accurate self-reporting of medication errors. More prospective and observational research is needed to determine the differences in actual versus self-reported medical errors as well determine if cultural differences affect the rate of self-reported medical errors. Many hospitals in the U.S. do not have a standardized transition program for IENs (Baptise, 2015). Transition program that includes nursing orientation and social support; understanding of the scope of practice, policies, and procedures in the U.S. may help IENs adapt to the U.S. work environment easily (Woltcott et al. 2013). Developing a transition program into the U.S. work environments for IENs may also help reduce stress especially during their first year of employment (Xu and He, 2012).

From a policy perspective, standardizing new employee orientation may be inadequate for IENs to integrate into the U.S. health care delivery system. Policies that encourage targeted orientation addressing implicit belief systems about the nursing role and explains patient safety expectations as well as procedures for medication administration may be particularly beneficial for IENs. Supportive leadership that is culturally competent, ensures ongoing continuing education in pharmacology, and provides culturally appropriate incentives for self-reporting medication errors are important. Finally, policies that provide decentralized pharmacists to observe medication administration provide in services on medication administration, and liaison with patient care providers in high acuity units has been shown to reduce medication errors.

This study has limitations. First, the results of our study should be interpreted cautiously. Our sample was limited to IENs and USENs who practiced in the southwest region of the U.S. which limits the generalizability of our findings. In addition, the five participating hospitals were with three different hospital systems where the format of recording medication error incidents as well as other data systems at their respective risk management departments varied. For instance, the nurses in the control group were identified through the pharmacy department at the two hospital systems while the nurses in the control group were identify through the human resource database at one hospital system where the match approach to the case group was different. Finally, except for 
IENs from Philippine, the numbers of IENs from other countries in our sample were too small for us to further examine potential variations in making medication errors among subgroups of IENs.

\section{ACKNOWLEDGEMENT}

This research project was funded by the National Council of State Boards of Nursing (Grant No. R40006-2). Correspondence regarding this article should be sent to Jay Shen, Ph.D., Department of Health Care Administration and Policy, University of Nevada Las Vegas, e-mail: jay.shen@unlv.edu

\section{REFERENCES}

Baptiste, M. (2015). Workplace discrimination: An additional stressor for internationally educated nurses. OJIN: The Online Journal of Issues in Nursing, 20(3). https:/ / doi.org/10.3912/OJIN.Vol20No03PPT01

Casey, T. W., Riseborough, K. M. and Krauss, A. D. (2015). Do you see what I see? Effects of national culture on employees' safety-related perceptions and behavior. Accident Analysis \& Prevention, 78, 173-184. https://doi.org/10.1016/j.aap.2015.03.010

Hayes, L. J., O’Brien-Pallas, L., Duffield, C., Shamian, J., Buchan, J., Hughes, F.,... North, N. (2012). Nurse turnover: A literature review - an update. International Journal of Nursing Studies, 49(7), 887-905. https://doi.org/10.1016/j.ijnurstu.2011.10.001

Keers, R. N., Williams, S. D., Cooke, J. and Ashcroft, D. M. (2013). Causes of medication administration errors in hospitals: a systematic review of quantitative and qualitative evidence. Drug safety, 36(11), 1045-1067. https://doi.org/10.1007/s40264-013-0090-2

Marx, M. (2014). Examining the structural challenges to communication as experienced by nurse managers in two US hospital settings. Journal of nursing management, 22(8), 964-973. https://doi.org/10.1111/jonm.12091

Neff, D. F. and Harman, J. (2013). Foreign-educated nurses: Effects on nurse, quality of care, and patient-safetyindicator outcomes. Journal of Nursing Regulation, 4(1), 19-24. https:/ / doi.org/10.1016/S2155-8256(15)30163-0

O’Byrne, N., Kozub, E. I. and Fields, W. (2016). Reducing Continuous Intravenous Medication Errors in an Intensive Care Unit. Journal of Nursing Care Quality, 13-16. https:// doi.org/10.1097/NCQ.0000000000000144

Parry, A. M., Barriball, K. L. and While, A. E. (2015). Factors contributing to Registered Nurse medication administration error: A narrative review. International journal of nursing studies, 52(1), 403-420. https://doi.org/10.1016/j.ijnurstu.2014.07.003

Patanwala, A. E., Warholak, T. L., Sanders, A. B. and Erstad, B. L. (2010). A prospective observational study of medication errors in a tertiary care emergency department. Annals of emergency medicine, 55(6), 522-526. https://doi.org/10.1016/j.annemergmed.2009.12.017

Shen, J. J., Neff, D. F., Cimiotti, J., Sloane, D. M. and Aiken, L. H. (2013). Utilization of non-US educated nurses in US hospitals: Implications for hospital mortality. International Journal for Quality in Health Care, 366-372.

Shen, J. J., Xu, Y., Bolstad, A., Covelli, M., Torpey, M., Colosimo, R. and Jorgenson, M. (2012). Effects of a shortterm linguistic class on communication competence of international nurses: Implications for practice, policy, and research. Nursing Economics, 30(1), 21-8.

Shen, J. J., Xu, Y., Staples, S. and Bolstad, A. (2014). Using the Interpersonal Skills Tool to Assess Interpersonal Skills of Internationally Educated Nurses. Japan Journal of Nursing Science, 11(3), 171-9. https://doi.org/10.1111/jjns.12018

Shen, J. J., Neishi, S., VanBeuge, S., Covelli, M., Adamek, S., Gallegos, J. and Gardner, M. R. (2015). Comparing Medication Error Incidents among Foreign-Educated Nurses and US-Educated Nurses. Journal of Nursing Regulation, 5(4), 4-10. https://doi.org/10.1016/S2155-8256(15)30030-2

Sherwood, G. D. and Shaffer, F. A. (2014). The role of internationally educated nurses in a quality, safe workforce. Nursing outlook, 62(1), 46-52. https://doi.org/10.1016/j.outlook.2013.11.001

U. S. Food and Drug Adminiatration. (2016). Medication Error Reports. Available at: http://www.fda.gov/Drugs/DrugSafety/MedicationErrors/ucm080629.htm (Accessed 28 December 2016)

Wagner, L. M., Brush, B. L., Engberg, J. B., Castle, N. G. and Capezuti, E. (2015). Quality care outcomes in nursing homes: The effects of a nurse's country of origin and education. Journal of Nursing Regulation, 5(4), 49-56. https://doi.org/10.1016/S2155-8256(15)30032-6

Watt-Watson, J., Stevens, B., Garfinkel, P., Streiner, D. and Gallop, R. (2001). Relationship between nurses' pain knowledge and pain management outcomes for their postoperative cardiac patients. Journal of Advanced Nursing, 36(4), 535-545. https:/ / doi.org/10.1046/j.1365-2648.2001.02006.x

Weant, K. A., Bailey, A. M. and Baker, S. N. (2014). Strategies for reducing medication errors in the emergency department. Open access emergency medicine: OAEM, 6, 45. https://doi.org/10.2147/OAEM.S64174 
Wolcott, K., Llamado, S. and Mace, D. (2013). Integration of internationally educated nurses into the U.S. Workforce. Journal of Nurses in Professional Development, 29(5), 263-268. https://doi.org/10.1097/01.NND.0000433145.43933.98

Xu, Y., Shen, J. J., Bolstad, A. L., Covelli, M. and Torpey, M. (2010). Evaluation of an Intervention on SocioCultural Communication Skills of International Nurses. Nursing Economics, 28(6), 386-392.

$\mathrm{Xu}, \mathrm{Y}$. and He, F. (2012). Transition programs for internationally educated nurses: What can the United States learn from the United Kingdom, Australia, and Canada? Nursing Economics, 30(4), 215-223, 239.

$\mathrm{Xu}$, Y., Zaikina-Montgomery, H. and Shen, J. J. (2010). Characteristics of internationally educated nurses in the United States: An update from the 2004 National Sample Survey of Registered Nurses. Nursing Economics, 28(1), 19. 